\title{
Does PCNA diffusion on DNA follow a rotation- coupled translation mechanism?
}

\author{
Harry Mark Greenblatt ${ }^{1}$, Haim Rozenberg${ }^{1}$, Dina Daitchman ${ }^{1} \&$ Yaakov Levy $\mathbb{1}^{1 凶}$ \\ ARISING From De March et al. Nature Communications https://doi.org/10.1038/ncomms13935 (2017)
}

$\mathrm{V}$ arious DNA-binding proteins were shown, by both experimental and theoretical approaches, to diffuse in a rotationcoupled translation manner ${ }^{1-4}$, while the mechanistic features of this sliding dynamics may vary for different proteins depending on their molecular properties ${ }^{1,4-7}$. In the work by De March et al. ${ }^{8}$, using three different techniques (X-ray crystallography, nuclear magnetic resonance (NMR) measurements, and molecular dynamics (MD) simulations), the authors claim that the toroidal PCNA protein also diffuses along DNA in a motion that is coupled to rotation along the DNA axis. We found that the NMR and MD reported by De March et al. to be inconclusive, and, most importantly, the diffraction data do not support the reported location of the DNA. In light of this, the proposed spiral cog-wheel mechanism, where the clamp keeps a fixed orientation relative to the DNA, as the main component for PCNA diffusion along DNA, is not supported by their work.

De March et al. present a crystal structure of a complex between the homotrimer of human PCNA and a $10 \mathrm{bp}$ stretch of double stranded DNA (originally deposited as PDB 5L7C) ${ }^{8}$. In their model, they identify five interactions between arginine or lysine side chains and the DNA phosphate backbone. These unique interactions are used by the authors to propose that "the orientation of the clamp is invariant relative to DNA" and the PCNA-DNA interface thus supports "a helical sliding mechanism in which the clamp rotates and tilts by keeping a fixed orientation relative to the DNA backbone"s. Examining the deposited structure and data, however, we felt that the purported interactions between the protein and the DNA were not supported by the diffraction data, due to extremely high atomic thermal displacement parameters (B-factors) for the DNA duplex, and poor density on the DNA. During our correspondence with the authors while this communication was in preparation, they revised the PDB file (6GIS), indicating that the original submitted version of 5L7C was in error. Our remarks, therefore, are based on this revised coordinate file. In the modified PDB file the average B-factor for the DNA is indeed lower, yet is still $~ 3.4$ times higher relative to that of the protein. Furthermore, the average B-factors for the side-chains of the protein residues that the authors show interacting with the DNA are not lower than their counterparts in other chains. If specific residues of a given subunit are interacting with a ligand, one expects their B-factors to be lower than the equivalent free residues in other subunits due to these stabilizing interactions. This, however, is not the case: the side-chain B-factors of Lys20, Lys217, Lys80, and His153 are similar in all three subunits. In fact, Lys77 and Arg149 of chain A appear even more disordered than their counterparts in other chains. It should also be noted that Arg149 of Chain A does not even form a salt bridge with the DNA, since its polar end is facing away from the DNA backbone. The closest polar atom of the side chain of Arg149 is $\mathrm{N} \varepsilon$, at $4.2 \AA$ from the closest phosphate oxygen, too far for a standard H-bond.

Finally, and most importantly, we could not see any difference density ( $\mathrm{mFo}-\mathrm{DFc})$ in an omit map that could unambiguously locate the DNA in the position reported by the authors. Examining the difference maps (even at $1.3 \sigma$, as used by De March et al.), we see no density characteristic of B-DNA, such as basepair stacking or phosphate backbone (Fig. 1a-c). In fact, at $1.3 \sigma$, there is so much noise, one must ask why the authors decided to place the DNA where they did, while ignoring the rest of the copious density. It may be argued that standard solvent modeling would eliminate the weak density for some low occupancy DNA. To investigate this possibility, we used Phenix to generate a Polder omit map 9 . In Fig. 1c, we show the results of the Polder map; even if one could argue that there is some structural signal, it is at low occupancy and not recognizable as DNA.

As a control, we performed a similar analysis using the E. coli sliding clamp on DNA (3BEP) $)^{10}$ by removing DNA from the structure and running simulated annealing. In the resultant difference map (Fig. 1d), despite the poor quality of the density, at $2 \sigma$ the distinctive shape of stacked bases is clear, indicating the presence of DNA. This is in contrast to the results for 6GIS.

Given these observations, placement of duplex DNA at its published location, especially at $100 \%$ occupancy, is therefore not justified by the X-ray crystallographic analysis of De March et al. ${ }^{8}$ As such, their claims about specific residues interacting with the DNA have no structural basis. De March et al. comment on "the existence of a subpopulation of complexes with slightly different DNA orientations" to explain the high thermal parameters and the potential exchange between symmetric sites of PCNA. This uncertainty, however, in the DNA orientation is highly 

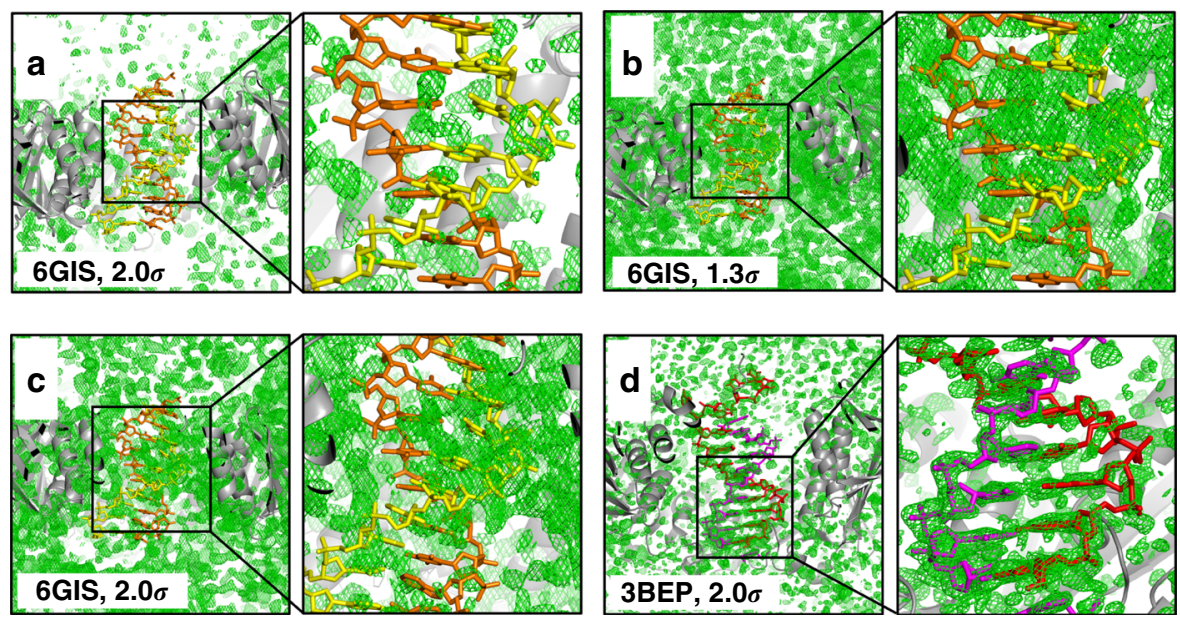

Fig. 1 Assessment of the electron density of putative DNA in PDB 6GIS. a Simulated annealing difference omit map (mFo-DFc), contoured at $2.0 \sigma$, overlaid with the model of DNA from the crystal structure, showing no evidence of DNA bound to the interior of PCNA. The DNA strand that interacts with the protein is colored yellow, and the complementary strand is colored orange. $\mathbf{b}$ Same difference map at $1.3 \sigma$. Note that the noisy density also covers parts of the protein, indicating that the contour level is too low, but still does not correspond to the DNA. c Polder omit map contoured at $1.3 \sigma$, showing copious density, but not corresponding to DNA, particularly on the lower left of the yellow strand, which purportedly interacts with PCNA. In order to generate an effective omit map, we removed the DNA (7.2\% of the total atoms), and ran simulated annealing using PHENIX ${ }^{11}$ to decrease bias from the starting model. We also removed all TLS and NCS parameters, and used group B factors (2 values per residue, in consideration of the relatively low resolution: $2.82 \AA$ ), but no other adjustments were made to the model. This resulted in a significant drop in both Rwork (from 24.7 to $19.5 \%$ ) and Rfree (from 28.4 to $24.9 \%$ ), and the final geometry was also improved (RMSD bonds from 0.016 to $0.008 \AA$, and RMSD angles from 1.8 to $1.0^{\circ}$ ), clearly indicating a large improvement in the model. The RMSD between the starting structure and the final structure was $0.51 \AA$ for all protein atoms. In order to confirm that the improvement in Rwork and Rfree were not resulting from differences between Phenix and REFMAC ${ }^{12}$, the output from Phenix was run through REFMAC, giving a final Rwork of $19.1 \%$, and Rfree of $24.6 \%$. d Simulated annealing difference omit map, for 3BEP, contoured at $2 \sigma$. Images made in PyMOL.

underestimated, and should at least be reflected in significantly lower occupancy for the DNA. Furthermore, exchange between symmetrical sites on the three PCNA subunits can hardly be termed "slightly different" orientations.

The NMR data show interactions between the residues that line the inner ring of PCNA and the DNA, but these observations are consistent with interactions that are distributed symmetrically around the inner ring. These interactions could be formed simultaneously by centrally located DNA, by transient interactions as the DNA moves around the inner channel, or a combination of both. This would allow for simple linear translation of the DNA, without requiring rotation. All these mechanisms are governed by transient PCNA-DNA interactions which are consistent with the very high $K_{\mathrm{D}}(\sim 0.7 \mathrm{mM})$.

The MD simulations based on the 5L7C hPCNA-DNA complex suggests that the five interfacial salt-bridges are formed throughout the $250 \mathrm{~ns}$ simulation, but this might be a direct consequence of using the biased experimental model as the initial structure for the simulations. No direct diffusion, however, is shown in this simulation, most probably because of the short timescale, rendering their results inconclusive ${ }^{8}$.

Coarse-grained molecular dynamics simulations and electrostatic free energy calculations support a weak PCNA-DNA interface and therefore one-dimensional diffusion of PCNA along DNA should occur via translocation that is uncoupled from rotation where the DNA is placed, on average, at the center of the PCNA inner ring ${ }^{13}$. Obviously, direct interactions between PCNA and DNA are, in principle, possible but they are expected to be transient and not lead to sustained helical motion of PCNA along DNA. Further support for uncoupled diffusion of PCNA on DNA comes from a singlemolecule experiment on the dependence of the diffusion coefficient of PCNA on its dimension ${ }^{14}$, which is very similar to that of the TALE protein that was concluded to follow uncoupled rotationtranslation diffusion along DNA ${ }^{15}$. The former single-molecule study, however, reported that upon changing the solvent viscosity
PCNA may track the DNA helical pitch ${ }^{14}$. These contradictory results, which were seemingly resolved following simple modeling by favoring the coupled rotation-translation diffusion of PCNA along DNA, demand further investigation of PCNA, as well as other toroidal proteins, to resolve the unique properties of their interactions with DNA and their ambiguous characteristics.

In conclusion, the proposed mechanism of translation-coupled rotation as the main mode of PCNA sliding along the DNA backbone, implies sustained contacts with the DNA by five residues of one single subunit, with stochastic exchange among adjacent phosphate atoms. This mechanism is at odds with the lack of electron density for DNA in the crystal structure, and the observed low binding affinity of PCNA for DNA. Furthermore, the lack of rotation in the MD simulations, and the inconclusive NMR data do not support this mechanism. While using complementary approaches to provide a fuller and consistent picture is commendable, we do not find that any of the results presented by De March et al. support the contention that PCNA slides in a rotation-coupled fashion.

\section{Data availability}

No datasets were generated for this manuscript.

Received: 30 May 2018; Accepted: 29 June 2020; Published online: 05 October 2020

\section{References}

1. Givaty, O. \& Levy, Y. Protein sliding along DNA: dynamics and structural characterization. J. Mol. Biol. 385, 1087-1097 (2009).

2. Blainey, P. C. et al. Nonspecifically bound proteins spin while diffusing along DNA. Nat. Struct. Mol. Biol. 16, 1224-1229 (2009).

3. Schurr, J. M. One-dimensional diffusion-coefficient of proteins absorbed on DNA-hydrodynamic considerations. Biophys. Chem. 9, 413-414 (1979). 
4. Iwahara, J. \& Clore, G. M. Detecting transient intermediates in macromolecular binding by paramagnetic NMR. Nature 440, 1227-1230 (2006).

5. Marcovitz, A. \& Levy, Y. Frustration in protein-DNA binding influences conformational switching and target search kinetics. Proc. Natl Acad. Sci. USA 108, 17957-17962 (2011).

6. Terakawa, T., Kenzaki, H. \& Takada, S. p53 Searches on DNA by rotationuncoupled sliding at $\mathrm{C}$-terminal tails and restricted hopping of core domains. J. Am. Chem. Soc. 134, 14555-14562 (2012).

7. Khazanov, N. \& Levy, Y. Sliding of p53 along DNA can be modulated by its oligomeric state and by cross-talks between its constituent domains. J. Mol. Biol. 408, 335-355 (2011).

8. De March, M. et al. Structural basis of human PCNA sliding on DNA. Nat Commun. 8, 13935 (2017).

9. Liebschner, D. et al. Polder maps: improving OMIT maps by excluding bulk solvent. Acta Crystallogr. Sect. D 73, 148-157 (2017).

10. Georgescu, R. E. et al. Structure of a sliding clamp on DNA. Cell 132, 43-54 (2008).

11. Adams, P. D. et al. PHENIX: a comprehensive python-based system for macromolecular structure solution. Acta Crystallogr. Sect. D 66, 213-221 (2010).

12. Murshudov, G. N. et al. REFMAC5 for the refinement of macromolecular crystal structures. Acta Crystallogr. Sect. D 67, 355-367 (2011).

13. Daitchman, D., Greenblatt, H. M. \& Levy, Y. Diffusion of ring-shaped proteins along DNA: case study of sliding clamps. Nucleic acids Res. 46, 5935-5949 (2018).

14. Kochaniak, A. B. et al. Proliferating cell nuclear antigen uses two distinct modes to move along DNA. J. Biol. Chem. 284, 17700-17710 (2009).

15. Cuculis, L., Abil, Z., Zhao, H. M. \& Schroeder, C. M. TALE proteins search DNA using a rotationally decoupled mechanism. Nat. Chem. Biol. 12, 831 (2016).

\section{Acknowledgements}

This work was supported by the Kimmelman Center for Macromolecular Assemblies and the Minerva Foundation. Y.L. holds The Morton and Gladys Pickman professional chair in Structural Biology.

\section{Author contributions}

H.M.G and H.R. analysed the crystal structure and the electron density derived from the X-ray diffraction data. H.M.G., H.R., D.D. and Y.L. wrote the manuscript.

\section{Competing interests}

The authors declare no competing interests.

\section{Additional information}

Correspondence and requests for materials should be addressed to Y.L.

Peer review information Nature Communications thanks the anonymous reviewers for their contribution to the peer review of this work.

Reprints and permission information is available at http://www.nature.com/reprints

Publisher's note Springer Nature remains neutral with regard to jurisdictional claims in published maps and institutional affiliations.

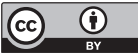

Open Access This article is licensed under a Creative Commons Attribution 4.0 International License, which permits use, sharing, adaptation, distribution and reproduction in any medium or format, as long as you give appropriate credit to the original author(s) and the source, provide a link to the Creative Commons license, and indicate if changes were made. The images or other third party material in this article are included in the article's Creative Commons license, unless indicated otherwise in a credit line to the material. If material is not included in the article's Creative Commons license and your intended use is not permitted by statutory regulation or exceeds the permitted use, you will need to obtain permission directly from the copyright holder. To view a copy of this license, visit http://creativecommons.org/ licenses/by/4.0/.

(C) The Author(s) 2020 